14,15

\title{
Формирование вихревых течений в жидкокристаллических фазах инкапсулированных в микролитровые объемы под действием сфокусированного лазерного излучения
}

\author{
(C) A.B. Захаров \\ Институт проблем машиноведения РАН, \\ Санкт-Петербург, Россия \\ E-mail: alexandre.zakharov@yahoo.ca
}

(Поступила в Редакцию 17 января 2018 г.)

\begin{abstract}
Предложено теоретическое описание процесса формирования вихревых течений $\mathbf{v}(t, \mathbf{r})$ и эволюции поля директора $\hat{\mathbf{n}}$ в микролитровых жидкокристаллических (ЖК) объемах со свободной поверхностью под действием градиента температуры $\nabla T(t, \mathbf{r})$, инициируемого сфокусированным лазерным излучением. В рамках нелинейного обобщения классической теории Эриксена-Лесли учтены термомеханические вклады как в тензор напряжений, так и в вязкий момент, действующие на единицу объема ЖК-фазы, что позволило описать зарождение и формирование вихревых потоков в нематиках, образованных молекулами 4-н-пентил-4'-цианобифенила. Численными методами были исследованы различные гидродинамические режимы формирования вихрей в микроразмерных ЖК-объемах под действием сфокусированного лазерного излучения.
\end{abstract}

Работа выполнена при финансовой поддержке РФФИ (грант № 16-02-00041a).

DOI: 10.21883/FTT.2018.07.46135.011

\section{1. Введение}

Наряду с широким использованием жидких кристаллов (ЖК) в производстве ЖК-дисплеев другой не менее интригующей областью применения ЖК является микро- и нанофлуидистика, т. е., наука о движении молекулярной жидкости в микро- и наноразмерных каналах и капиллярах. Молекулярные жидкости инкапсулированные в микро- и нанолитровые объемы находят применение в разнообразных сенсорах и датчиках используемых в биотехнологических приложениях, медицине и фармокологии, а также в иммерсионной литографии. Методы микро- и нанофлуидистики находят также применение при исследовании процессов транспортировки и сортировки нанолитровых капель жидкости и ЖК в разветвленных каналах и капиллярах (lab-on-chip-system) под действием электрического поля (электрокинетика) [1]. Еще один из путей манипулирования такими молекулярными системами - это формирование градиентов поверхностного натяжения (ГПН) на границах раздела жидкость/газ и жидкость/твердое тело [2]. Было показано, что разность ГПН инициируемая локализованным разогревом способна привести в движение микролитровые капли молекулярной жидкости в микроразмерных капиллярах [3]. Также недавно был предложен новый метод транспортировки микролитровых ЖК-капель инкапсулированных в микроразмерные каналы и капилляры под действием градиента температуры, создаваемого за счет разности температур на ограничивающих поверхностях $[4,5]$. А принимая во внимание тот факт, что такая разновидность ЖК-систем, как лиотропные ЖК, составляют основу многих сложных биологических ор- ганизмов [6,7], то возможность манипулирования такими биосистемами посредством локального формирования градиентов температуры открывает новые перспективы в биотехнологических приложениях. В свою очередь, локальный разогрев микроразмерной ЖК-системы (ЖК-капельки) возможен с помощью лазерного излучения сфокусированного как в объеме ЖК-фазы [3,5], так и на свободной границе раздела ЖК-фаза/воздух [8]. Наличие свободной ограничивающей поверхности в таких ЖК-системах влияет на характер переориентации поля директора и формирование вихревых потоков под действием сфокусированного лазерного излучения [9]. Было показано, что основным физическим механизмом ответственным за возникновение гидродинамических потоков в ЖК-системах инкапсулированных в микроразмерные каналы и капилляры является взаимодействие градиентов поля директора ЖК-фазы и температуры $[4,10]$. Если локальный градиент температуры в объеме такой ЖК-фазы легко формируется с помощью сфокусированного лазерного излучения, то градиент поля директора удается сформировать посредством гибридной ориентации ЖК-фазы на ограничивающих поверхностях. В случае ЖК-фазы со свободной поверхностью на границе раздела ЖК-фаза/воздух всегда формируется гомеотропная ориентация молекул образующих ЖК-систему [11]. Таким образом, гибридная ориентация поля директора внутри микролитровой ЖК-ячейки со свободной границей раздела может быть достигнута посредством планарной ориентации молекул ЖК-фазы вдоль нижней и боковых ограничивающих поверхностей. Таким образом, все это указывает на то, что существует возможность немеханической транспортировки или сортировки мик- 
ролитровых объемов ЖК-фазы под действием сфокусированного лазерного излучения. Поэтому для того, чтобы эффективно манипулировать микроразмерными объемами жидкости (капельками), необходимо изучить влияние внешних сил как на структурные, так и на динамические свойства таких систем.

Целью нашего исследования является описание эволюции поля директора $\hat{\mathbf{n}}$ и формирование вихревых потоков $\mathbf{v}(t, \mathbf{r})$ в микролитровых ЖК-ячейках со свободной поверхностью под действием градиента температуры $\nabla T(t, \mathbf{r})$, инициируемого сфокусированным лазерным излучением. Эти исследования будут проведены в рамках нелинейного обобщения классической теории Эриксена-Лесли [12,13], с учетом не только баланса массы, импульсов и угловых моментов действующих на единицу объема ЖК-фазы, но и с учетом баланса энтропии [14]. При этом будут учтены термомеханические вклады как в полную диссипационную функцию Релея, так и в баланс моментов [4,10]. Численными методами будут исследованы различные гидродинамические режимы формирования вихрей в микроразмерных ЖК-каналах под действием сфокусированного лазерного излучения.

\section{2. Основные гидродинамические уравнения}

Рассмотрим длинную прямоугольную ЖК-ячейку с размерами $2 L$ и $2 d(L \gg d)$, ограниченную нижней твердой горизонтальной и двумя вертикальными поверхностями, в то время как сверху ЖК-фаза граничит с воздухом. Допустим, что директор $\hat{\mathbf{n}}=n_{x} \hat{\mathbf{i}}+n_{z} \hat{\mathbf{k}}$ все время находится в плоскости $X Z$ и при этом планарно ориентирован на нижней горизонтальной и двух вертикальных поверхностях и гомеотропно на свободной поверхности, граничащей с воздухом. Таким образом, мы имеем дело с гибридно-ориентированной ЖК(ГОЖК)-фазой, характеризующейся сильным сцеплением ЖК-молекул с ограничивающими поверхностями. В дальнейшем будем считать, что система координат отсчитывается от середины ЖК-ячейки так, что ось $X$ и орт $\hat{\mathbf{i}}$ совпадают с направлением директора на нижней горизонтальной поверхности $\left(\hat{\mathbf{i}} \| \hat{\mathbf{n}}_{z=-d}\right)$, в то время как ось $Z$ и орт $\hat{\mathbf{k}}$ направлены ортогонально $\left(\hat{\mathbf{k}} \perp \hat{\mathbf{n}}_{z=-d}\right)$, а орт $\hat{\mathbf{j}}=\hat{\mathbf{k}} \times \hat{\mathbf{i}}$. Таким образом, в объеме ЖК-фазы формируется градиент поля директора $\nabla \hat{\mathbf{n}}$. При этом верхняя, изначально горизонтальная свободная поверхность раздела ЖК фаза/воздух под действием лазерного излучения деформируется, и эта деформация характеризуется кривизной $\kappa$, а также нормалью $v$ и единичным касательным вектором $\hat{\mathbf{t}}$. Под действием лазерного излучения в исследуемой среде возникает градиент температуры $\nabla T$, который взаимодействует с градиентом поля директора $\nabla \hat{\mathbf{n}}$. Ранее нами было показано, что учет термомеханических вкладов как в тензор напряжений, так и в вязкий момент, действующих на единицу объема ЖК-фазы, приводит к формированию гидродинамических потоков в ЖК-ячейках [4]. Таким образом, вследствие формирования гидродинамического течения $\mathbf{v}$ в ЖК-ячейке под действием лазерного излучения происходит переориентация поля директора $\hat{\mathbf{n}}$, которая может быть описана в рамках обобщенной теории Эриксена-Лесли [12,13], учитывающей баланс массы, импульсов, угловых моментов и энтропии $[4,5]$. Принимая во внимание микроскопические размеры ЖК-канала мы можем предположить, что плотность $\rho$ ЖК-системы постоянна и мы имеем дело с несжимаемой жидкостью. Это позволяет нам записать уравнения сохранения в виде $[4,5,9]$

$$
\begin{gathered}
\nabla \cdot \mathbf{v}=0, \\
\mathbf{T}_{\mathrm{el}}+\mathbf{T}_{\mathrm{vis}}+\mathbf{T}_{\mathrm{tm}}=0, \\
\rho \frac{d \mathbf{v}}{d t}=\nabla \cdot \sigma,
\end{gathered}
$$

где $\mathbf{T}_{\mathrm{el}}=\frac{\delta \mathscr{W}_{\mathrm{el}}}{\delta \hat{\mathbf{n}}} \hat{\mathbf{n}}-$ упругий, $\mathbf{T}_{\mathrm{vis}}=\frac{\delta \mathscr{R}^{\mathrm{vis}}}{\delta \tilde{\mathbf{n}}_{t}} \hat{\mathbf{n}}-$ вязкий и $\mathbf{T}_{\mathrm{tm}}=\frac{\delta \mathscr{R}^{\mathrm{tm}}}{\delta \hat{\mathbf{n}}_{t}} \hat{\mathbf{n}}$ термомеханический вклады в баланс моментов действующих на единицу объема ЖК-фазы. Здесь $\quad \sigma=\sigma_{\mathrm{el}}+\sigma_{\mathrm{vis}}+\sigma_{\mathrm{tm}}+\sigma_{\mathrm{th}}-P \mathscr{E}-$ тензор напряжений, состоящий из упругого $\sigma^{\mathrm{el}}=-\frac{\partial \mathscr{W}_{\mathrm{el}}}{\partial \nabla \mathbf{n}}(\nabla \hat{\mathbf{n}})^{T}$, вязкого $\quad \sigma^{\text {vis }}=\frac{\delta \mathscr{R}^{\text {vis }}}{\delta \nabla \mathbf{v}}, \quad$ термомеханического $\quad \sigma^{\mathrm{tm}}=\frac{\delta \mathscr{R}^{\mathrm{tm}}}{\delta \nabla \mathbf{v}}$ и термического $\sigma^{\text {th }}=\frac{\delta \mathscr{R}^{\text {th }}}{\delta \nabla \mathbf{v}} \quad$ вкладов $\quad$ соответственно, $P$ - гидростатическое давление в ЖК-системе и $\mathscr{E}-$ единичный тензор. Выражение для полной диссипационной функции Релея имеет вид $\mathscr{R}=\mathscr{R}^{\mathrm{vis}}+\mathscr{R}^{\mathrm{tm}}+\mathscr{R}^{\mathrm{th}}, \quad$ в $\quad$ то время как плотность упругой энергии может быть записана в виде $2 \mathscr{W}_{\mathrm{el}}=K_{1}(\nabla \hat{\mathbf{n}})^{2}+K_{3}(\hat{\mathbf{n}} \nabla \hat{\mathbf{n}})^{2}, \quad$ где $\quad K_{1} \quad$ и $\quad K_{3} \quad-$ коэффициенты упругости Франка соответствующие поперечному и продольному изгибам. Выражение для вязкого вклада в диссипационную функцию Релея имеет вид $\quad \mathscr{R}^{\text {vis }}=\alpha_{1}\left(\hat{\mathbf{n}} \mathbf{D}_{s} \hat{\mathbf{n}}\right)^{2}+\gamma_{1}\left(\hat{\mathbf{n}}_{t}-\mathbf{D}_{a} \hat{\mathbf{n}}\right)^{2}+2 \gamma_{2}\left(\hat{\mathbf{n}}_{t}-\mathbf{D}_{a} \hat{\mathbf{n}}\right)$ $\times\left(\mathbf{D}_{s} \hat{\mathbf{n}}-\left(\hat{\mathbf{n}} \mathbf{D}_{s} \hat{\mathbf{n}}\right) \hat{\mathbf{n}}\right)+\alpha_{4} \mathbf{D}_{s}: \mathbf{D}_{s}+\left(\alpha_{5}+\alpha_{6}\right)\left(\hat{\mathbf{n}} \mathbf{D}_{s} \mathbf{D}_{s} \hat{\mathbf{n}}\right), \quad \mathbf{B}$ то время как термомеханический и термический вклады в диссипационную функцию Релея могут быть записаны в $\quad$ виде $\quad \frac{1}{\xi} \mathscr{R}^{\mathrm{tm}}=(\hat{\mathbf{n}} \nabla T) \mathbf{D}_{s}: \mathbf{M}+\nabla T \mathbf{D}_{s} \mathbf{M} \hat{\mathbf{n}}+(\hat{\mathbf{n}} \nabla T)$ $\times\left(\hat{\mathbf{n}}_{t}-\mathbf{D}_{a} \hat{\mathbf{n}}-3 \mathbf{D}_{s} \hat{\mathbf{n}}+3\left(\hat{\mathbf{n}} \mathbf{D}_{s} \hat{\mathbf{n}}\right) \hat{\mathbf{n}}\right) \mathbf{M} \hat{\mathbf{n}}+\hat{\mathbf{n}}(\nabla \mathbf{v})^{T} \mathbf{M} \nabla T$ $+\frac{1}{2}\left(\hat{\mathbf{n}} \mathbf{D}_{s} \hat{\mathbf{n}}\right) \nabla T \mathbf{M} \hat{\mathbf{n}}+\hat{\mathbf{n}}_{t} \mathbf{M} \nabla T+\frac{1}{2} \mathscr{M}_{0} \nabla T \nabla \mathbf{v} \hat{\mathbf{n}}+(\hat{\mathbf{n}} \nabla T) \mathscr{M}_{0}$ $\times\left(\hat{\mathbf{n}} \mathbf{D}_{s} \hat{\mathbf{n}}\right)+\frac{1}{2} \mathscr{M}_{0} \hat{\mathbf{n}}_{t} \nabla T \quad$ и $\quad \mathscr{R}^{\text {th }}=\frac{1}{T}\left(\lambda_{\|}(\hat{\mathbf{n}} \nabla T)\right)^{2}+\lambda_{\perp}$ $\times\left(\nabla T-\hat{\mathbf{n}}(\hat{\mathbf{n}} \nabla T)^{2}\right) \quad$ соответственно. Здесь $\alpha_{1}-\alpha_{6}-$ коэффициенты вязкости Лесли, $\gamma_{1}$ и $\gamma_{2}-$ коэффициенты вращательной и сдвиговой вязкости соответственно, $\xi-$ термомеханическая постоянная, а $\lambda_{\|}$и $\lambda_{\perp}-$ коэффициенты теплопроводности ЖК-фазы, соответствующие параллельному и перпендикулярному направлению директора соответственно. Симметричный и антисимметричный вклады в тензор градиента скорости принимают вид $2 \mathbf{D}_{s}=\nabla \mathbf{v}+(\nabla \mathbf{v})^{T} \quad$ и $\quad 2 \mathbf{D}_{a}=\nabla \mathbf{v}-(\nabla \mathbf{v})^{T}$ соответственно, тензор $2 \mathbf{M}=\nabla \hat{\mathbf{n}}+(\nabla \hat{\mathbf{n}})^{T}$, и $\mathscr{M}_{0}=\nabla \hat{\mathbf{n}}-$ скалярный инвариант тензора М. Здесь символ $T$ означает транспонирование матрицы, соответствующей $\nabla \mathbf{v}$. 
В дальнейшем будем исследовать гидродинамические режимы $\nabla T \sim 1 \mathrm{~K} / \mu \mathrm{m}$, возникающие в ЖК-канале микронных размеров под действием сфокусированного лазерного излучения через верхнюю свободную поверхность. При этом поле температуры $T(x, z, t)$ удовлетворяет уравнению теплопроводности $[4,5,9]$

$$
\rho C_{P} \frac{d T}{d t}=-\nabla \mathbf{q}+\mathscr{O}(x, z),
$$

где вектор $\mathbf{q}=-T \frac{\delta \mathscr{R}}{\delta \nabla T}$ представляет собой тепловой поток в ЖК-систему, а $C_{P}$ - теплоемкость ЖК-фазы, $\mathcal{O}(x, z)=\mathscr{O}_{0} \exp \left[-2 \frac{\left(x-x_{0}\right)^{2}+\left(z-z_{0}\right)^{2}}{\Delta^{2}}\right] \mathscr{H}\left(t_{\text {in }}-t\right)-$ плотность внутренних источников тепла $[9], \mathscr{H}\left(t_{\text {in }}-t\right)-$ функция Хевисайда, $\mathscr{O}_{0}=\frac{2}{\pi} \frac{\alpha \mathscr{V}_{0}}{\Delta^{2}}-$ коэффициент теплового потока, $\alpha-$ коэффициент абсорбции, $\mathscr{V}_{0}-$ мощность лазерного излучения, $\Delta-$ размер Гауссового пятна лазерного излучения, $t_{\text {in }}-$ продолжительность накачки лазерной энергии в ЖК-образец, а $x_{0}$ и $z_{0}-$ координаты центра лазерного пятна.

При этом будем считать, что температура на всех твердых ограничивающих поверхностях постоянна и равна

$$
T_{-L<x<L, z=-d}=T_{x= \pm L,-d<z<d}=T_{0},
$$

в то время как через верхнюю свободную поверхность отсутствует поток тепла, так что граничное условие на свободной поверхности может быть записано в виде

$$
\left(\mathbf{q}_{z=H} \cdot \boldsymbol{v}\right)=0,
$$

где функция $H(t, x)$ описывает деформацию свободной поверхности в процессе прогревания ЖК-канала. Поле скорости $\mathbf{v}=u \hat{\mathbf{i}}+w \hat{\mathbf{k}}$, образованное горизонтальной $u \equiv v_{x}(x, z, t)$ и вертикальной $w \equiv v_{z}(x, z, t)$ составляющими, подчиняется условию прилипания на твердых ограничивающих поверхностях

$$
\mathbf{v}_{-L<x<L, z=-d}=\mathbf{v}_{x= \pm L,-d<z<d}=0,
$$

в то время как условие на свободной поверхности связывает три независимых компоненты $\left(u_{, x}\right)_{z=H},\left(u_{, z}\right)_{z=H}$ и $(w, x)_{z=H}$ градиента поля скорости несжимаемой жидкости, где $u_{z}=\frac{\partial u}{\partial z}$. Эти компоненты определяются из условия баланса угловых моментов и сил на поверхности $z=H$, что позволяет нам записать эти балансы в виде

$$
\begin{gathered}
\left(\mathbf{T}_{\mathrm{el}}+\mathbf{T}_{\mathrm{vis}}+\mathbf{T}_{\mathrm{tm}}\right)_{z=H}=0, \\
\boldsymbol{v} \cdot \sigma_{z=H} \cdot \boldsymbol{v}=\gamma \kappa, \\
\boldsymbol{v} \cdot \sigma_{z=H} \cdot \hat{\mathbf{t}}=0 .
\end{gathered}
$$

Здесь $\gamma$ - коэффициент поверхностного натяжения свободной ЖК-поверхности. Зная функцию $z=H(t, x)$, мы можем определить нормальный $v=\left[-\frac{H_{, x}}{\sqrt{H_{, x}^{2}+1}}, 1\right]$ и касательный $\hat{\mathbf{t}}=\left[1, \frac{H_{, x}}{\sqrt{H_{, x}^{2}+1}}\right]$ векторы к этой поверхности, а также ее кривизну $\kappa=-\frac{H_{. x x}}{\sqrt{H_{, x}^{2}}+1}$ и ориентацию директора на поверхности $\hat{\mathbf{n}}_{z=H}=-\boldsymbol{v}$.
Уравнение описывающее деформацию свободной поверхности $H(x, t)$, вызванную локальным разогревом ЖК-материала, принимает вид

$$
\frac{\partial H}{\partial t}=w_{z=H}-u_{z=H} H_{, x},
$$

где $u_{z=H} \quad$ и $w_{z=H}$ горизонтальная и вертикальная компонента вектора скорости $\mathbf{v}$ на границе раздела ЖК-фаза/воздух соответственно. Система безразмерных уравнений, описывающих эволюцию поля директора, скорости и температуры под действием источника тепла может быть записана в виде

$$
\begin{aligned}
& n_{z} n_{x, \tau}-n_{x} n_{z, \tau}=\delta_{1}\left[n_{z} \mathscr{M}_{0, x}-n_{x} \mathscr{M}_{0, z}+K_{31}\left(n_{z} f_{, z}+n_{x} f_{, x}\right)\right] \\
& -\frac{1}{2} \psi_{, x x}\left[1+\gamma_{21}\left(n_{x}^{2}-n_{z}^{2}\right)\right]-\frac{1}{2} \psi_{, z z}\left[1-\gamma_{21}\left(n_{x}^{2}-n_{z}^{2}\right)\right] \\
& +2 \gamma_{21} \psi_{, x z} n_{x} n_{z}+\psi_{, z} \mathscr{N}_{x}+\mathscr{N}_{z} \psi_{, x}+\delta_{2}\left(\chi_{, x} \mathscr{L}_{, x}+\chi_{, z} \mathscr{L}_{, z}\right) \text {, } \\
& \delta_{3} \psi_{, x z \tau}=a_{1} \psi_{, z z z z}+a_{2} \psi_{, x z z z}+a_{3} \psi_{, x x z z}+a_{4} \psi_{, x x x z} \\
& +a_{5} \psi_{, x x x x}+a_{6} \psi_{, z z z}+a_{7} \psi_{, x z z}+a_{8} \psi_{, x x z} \\
& +a_{9} \psi_{, x x x}+a_{10} \psi_{, z z}+a_{11} \psi_{, x z}+a_{12} \psi_{, x x}+\mathscr{F}, \\
& \chi_{, \tau}=\left[\chi_{, x}\left(\Lambda n_{x}^{2}+n_{z}^{2}\right)+(\Lambda-1) n_{x} n_{z} \chi_{, z}\right]_{, x} \\
& +\left[\chi_{, z}\left(\Lambda n_{z}^{2}+n_{x}^{2}\right)+(\Lambda-1) n_{x} n_{z} \chi_{, x}\right]_{, z}+\delta_{4} \chi\left(\nabla \frac{\partial \mathscr{R}^{t m}}{\partial \nabla \chi}\right) \\
& +\delta_{5} \mathcal{O}(x, z, \tau)-\psi_{, z} \chi_{, x}+\psi_{, x} \chi_{, z},
\end{aligned}
$$

где $\tau=\frac{t}{t_{T}}-$ безразмерное время, $t_{T}=\frac{\rho C_{p} d^{2}}{\lambda_{\perp}}-$ характерное время используемое для нормировки, $\gamma_{21}=\gamma_{2} / \gamma_{1}-$ отношение коэффициентов вращательной вязкости, $\bar{\psi}=\frac{t_{T}}{d^{2}} \psi-$ безразмерный аналог функции тока $\psi$ для поля скорости $\mathbf{v}=u \hat{\mathbf{i}}+w \hat{\mathbf{k}}=-\nabla \hat{\mathbf{j}} \psi, \chi(x, z, \tau)=$ $=T(x, z, \tau) / T_{\mathrm{NI}}-$ безразмерная температура, $T_{\mathrm{NI}}-$ температура фазового перехода нематик-изотропное состояние, $f=n_{x, z}-n_{z, x}, n_{z, \tau}=\frac{\partial n_{z}}{\partial \tau}, \mathscr{M}_{0}=\nabla \cdot \hat{\mathbf{n}}, \mathscr{N}_{z}=n_{z} n_{x, z}$ $-n_{x} n_{z, z}, \mathscr{L}_{x}=n_{x} n_{z, x}-\frac{3}{2} n_{z} n_{x, x}+\frac{1}{2} n_{x} n_{x, z}, \mathscr{L}_{z}=-n_{z} n_{x, z}$ $+\frac{3}{2} n_{x} n_{z, z}-\frac{1}{2} n_{z} n_{z, x}$, а $\bar{x}=\frac{x}{d}$ и $\bar{z}=\frac{z}{d}-$ безразмерные пространственные переменные. В системе уравнений (10)-(12) и в последующем изложении черта над безразмерной функцией тока $\psi$ и безразмерными пространственными переменными $x$ и $z$ опущена. Выражения для функций $\mathscr{F}=\left(\sigma_{x x}^{\mathrm{el}}+\sigma_{x x}^{\mathrm{tm}}\right.$ $\left.-\sigma_{z z}^{\mathrm{el}}-\sigma_{z z}^{\mathrm{tm}}\right)_{, x z}+\left(\sigma_{z x}^{\mathrm{el}}+\sigma_{z x}^{\mathrm{tm}}\right)_{, z z}-\left(\sigma_{x z}^{\mathrm{el}}+\sigma_{x z}^{\mathrm{tm}}\right)_{, x x}, \sigma_{i j}^{\mathrm{tm}}(i, j=$ $=x, z)$ и $\sigma_{i j}^{\text {el }}(i, j=x, z)$, а также коэффициентов $a_{i}$ $(i=1, \ldots, 12)$ приведены в Приложении. Система уравнений (10)-(12) характеризуется набором параметров $K_{31}=\frac{K_{3}}{K_{1}}, \gamma_{21}=\frac{\gamma_{2}}{\gamma_{1}}, \Lambda=\lambda_{\|} / \lambda_{\perp}, \delta_{1}=\frac{t_{T} K_{1}}{\gamma_{1} d^{2}}, \delta_{2}=\frac{\rho C_{p} T_{\mathrm{NI}}}{\lambda_{\perp}} \frac{\xi}{\gamma_{1}}$, $\delta_{3}=\frac{\rho d^{2}}{\gamma_{1} t_{T}}, \delta_{4}=\frac{\xi}{\lambda_{\perp} t_{T}}$ и $\delta_{5}=\frac{2 \alpha}{\pi \Delta^{2}} \frac{d^{2}}{\lambda_{\perp} T_{\mathrm{NI}}} \mathcal{O}_{0}$.

Переориентация, под действием градиента температуры $\nabla \chi$, поля директора и скорости в микролитровом 
ГОЖК-объеме ограниченным одной нижней, двумя вертикальными стенками и сверху, в начальный момент, плоской свободной поверхностью может быть описана системой нелинейных дифференциальных уравнений в частных производных $(10)-(12)$ и дополненной граничными и начальными условиями как для поля директора и функции тока, так и для поля температуры. Так, на твердых поверхностях эти условия могут быть записаны в виде

$$
\begin{gathered}
\left(n_{x}\right)_{x= \pm 10,-1 \leq z \leq 1}=0, \quad\left(n_{x}\right)_{-10 \leq x \leq 10, z=-1}=1, \\
\chi_{x= \pm 10,-1 \leq z \leq 1}=0.97, \quad \chi_{-10 \leq x \leq 10, z=-1}=0.97 \\
\left(\psi_{, x}\right)_{x= \pm 10,-1 \leq z \leq 1}=\left(\psi_{, z}\right)_{x= \pm 10,-1 \leq z \leq 1}=0 \\
\left(\psi_{, x}\right)_{-10 \leq x \leq 10, z=-1}=\left(\psi_{, z}\right)_{-10 \leq x \leq 10, z=-1}=0,
\end{gathered}
$$

в то время как на свободной границе раздела ЖК-фаза/воздух эти условия принимают вид

$$
(\mathbf{n} \nabla \chi)_{z=H}=0, \quad(\mathbf{n} \boldsymbol{v})_{\mathrm{z}=\mathrm{H}}=-1, \quad \hat{\mathbf{B}} \boldsymbol{\Psi}=\mathbf{C} .
$$

Последнее уравнение является результатом решения системы уравнений (8) относительно трех безразмерных компонент градиента поля скорости $\vec{\Psi}=\left(\psi_{, x x}, \psi_{, x z}, \psi_{, z z},\right)$. Выражение для матрицы $\mathscr{B}$ и вектора $\mathbf{C}$ даны в Приложении, а начальные условия могут быть записаны в виде

$$
\begin{gathered}
\hat{\mathbf{n}}(\tau=0, x, z)=\hat{\mathbf{n}}_{\mathrm{el}}(x, z), \quad \psi(\tau=0, x, z)=0, \\
\chi(\tau=0, x, z)=0.97 .
\end{gathered}
$$

В уравнении (15) $\hat{\mathbf{n}}_{\mathrm{el}}(x, z)$ есть распределение упругого гибридного поля директора, удовлетворяющее уравнению

$$
n_{z} \mathscr{M}_{0, x}-n_{x} \mathscr{M}_{0, z}+K_{31}\left(n_{z} f_{, z}+n_{x} f_{, x}\right)=0,
$$

и граничным условиям

$$
\begin{gathered}
\left(n_{x}\right)_{x= \pm 10,-1 \leq z \leq 1}=\left(n_{x}\right)_{-10 \leq x \leq 10, z=1}=0, \\
\left(n_{x}\right)_{-10 \leq x \leq 10, z=-1}=1 .
\end{gathered}
$$

Решение системы (10)-(12) с граничными и начальными условиями (14)-(17) следует дополнить условием для определения профиля деформированной границы раздела ЖК-фаза/воздух

$$
H_{, \tau}+\left(\psi_{, x}\right)_{z=H}+\left(\psi_{, z}\right)_{z=H} H_{, x}=0 .
$$

Дальнейший анализ был проведен для случая ЖК-системы образованной цианобифинилом 5ЦБ при температуре $300 \mathrm{~K}$ и плотности $\rho=10^{3} \mathrm{~kg} / \mathrm{m}^{3}$. При температуре $300 \mathrm{~K}$ значения коэффициентов Франка $K_{1}$ и $K_{3}$ были выбраны равными $8.7[\mathrm{pN}]$ и $10[\mathrm{pN}][15]$, в то время как значения коэффициентов $\gamma_{1}$ и $\gamma_{2}$ были выбраны равными $0.069[\mathrm{~Pa} \cdot \mathrm{s}]$ и $-0.083[\mathrm{~Pa} \cdot \mathrm{s}][16]$ соответственно. Значения величин шести коэффициентов Лесли (в $[\mathrm{Pa} \cdot \mathrm{s}][16])$ равны: $\alpha_{1} \sim-0.0066, \alpha_{2} \sim-0.076, \alpha_{3} \sim-0.007, \alpha_{4} \sim 0.072$, $\alpha_{5} \sim 0.048$ и $\alpha_{6} \sim-0.03$ соответственно. Для коэффициентов теплопроводности в ЖК-системе образованной цианобифинилом 5ЦБ были выбраны следующие значения (в $[\mathrm{W} / \mathrm{m} \cdot \mathrm{K}][17]): \lambda_{\|}=0.24$ и $\lambda_{\perp}=0.13$ соответственно, а величина теплоемкости равна $[18] C_{p} \sim 10^{3}[\mathrm{~J} / \mathrm{kg} \cdot \mathrm{K}]$. В дальнейших расчетах была выбрана следующая величина поверхностного натяжения на границе раздела ЖК-фаза/воздух [19] $\gamma \sim 0.02[\mathrm{~N} / \mathrm{m}]$, а величина коэффициента абсорбции $\alpha$, для случая лазерного излучения с длиной волны в $1061[\mathrm{~nm}]$, равна $8\left[\mathrm{~m}^{-1}\right]$ [20]. В наших расчетах толщина $2 d$ ЖК-ячейки равна $10[\mu \mathrm{m}]$, а величина термомеханической постоянной $\xi$ была оценена в [21] как $\sim 1 \mathrm{pK} / \mathrm{N}$. Таким образом, безразмерные параметры системы (10)-(12) имеют следующие значения: $\delta_{1} \sim 10^{-3}, \delta_{2} \sim 0.3, \delta_{3} \sim 10^{-6}$ и $\delta_{4} \sim 10^{-4}$. Принимая во внимание тот факт, что безразмерная температура $\chi$ находится в пределах $[0.97-1.0)$, величину параметра $\delta_{5}$ можно оценить как $\delta_{5} \sim 7.0$. Эта оценка $\delta_{5}=\frac{2 \alpha}{\pi \Delta^{2}} \frac{d^{2}}{\lambda_{\perp} T_{\mathrm{NI}}} \mathcal{O}_{0}$ основывается на том, что продолжительность лазерного импульса мощностью в $\mathscr{V}_{0} \sim 0.5 \mathrm{~W}$, была $t_{\text {in }} \sim 2.0 \mu \mathrm{s}$, а размер лазерного пятна был выбран равным $\Delta=0.5 \mu \mathrm{m}$. Далее, принимая во внимание тот факт, что $\delta_{3} \ll 1$, уравнение (11) может быть переписано в виде

$$
\begin{aligned}
a_{1} \psi_{, z z z z} & +a_{2} \psi_{, x z z z}+a_{3} \psi_{, x x z z}+a_{4} \psi_{, x x x z}+a_{5} \psi_{, x x x x} \\
& +a_{6} \psi_{, z z z}+a_{7} \psi_{, x z z}+a_{8} \psi_{, x x z}+a_{9} \psi_{, x x x} \\
& +a_{10} \psi_{, z z}+a_{11} \psi_{, x z}+a_{12} \psi_{, z z}+\mathscr{F}=0 .
\end{aligned}
$$

\section{3. Деформация свободной поверхности ЖК-канала и формирование вихревых потоков}

Процесс формирования вихревых потоков, а также переориентации поля директора и формирование градиента температуры в узком ЖК-канале под действием лазерного излучения интенсивностью $\mathscr{V}_{0}=0.5 \mathrm{~W}$ и продолжительностью $2.0 \mu$ s описывается системой нелинейных дифференциальных уравнений в частных производных (10), (11) и (19) с учетом граничных (13)-(14) и начальных (15) условий. Система уравнений была решена методом релаксации [22] и сеточным методом [23]. Был изучен режим прогревания ЖК-фазы лазерным излучением мощностью $\mathscr{V}_{0}=0.5 \mathrm{~W}$ сфокусированным вблизи границы раздела ЖК-фаза/воздух на глубину $x_{0}=0.0$, $z_{0}=0.93$, или $\sim 0.7 \mu \mathrm{m}$ вглубь ЖК-фазы от свободной поверхности и продолжительностью $t_{\text {in }} \sim 2.0 \mu \mathrm{s}$. В начальный момент времени, с помощью уравнения (16), граничного условия (17) и условия отсутствия скорости $u=w=0$, было рассчитано поле температуры соответствующее $\hat{\mathbf{n}}_{\mathrm{el}}(x, z)$, что позволило также рассчитать функцию $\mathscr{F}$, компоненты матрицы $\mathscr{B}_{i j}$ и вектора С. Располагая начальными распределениями поля директора 

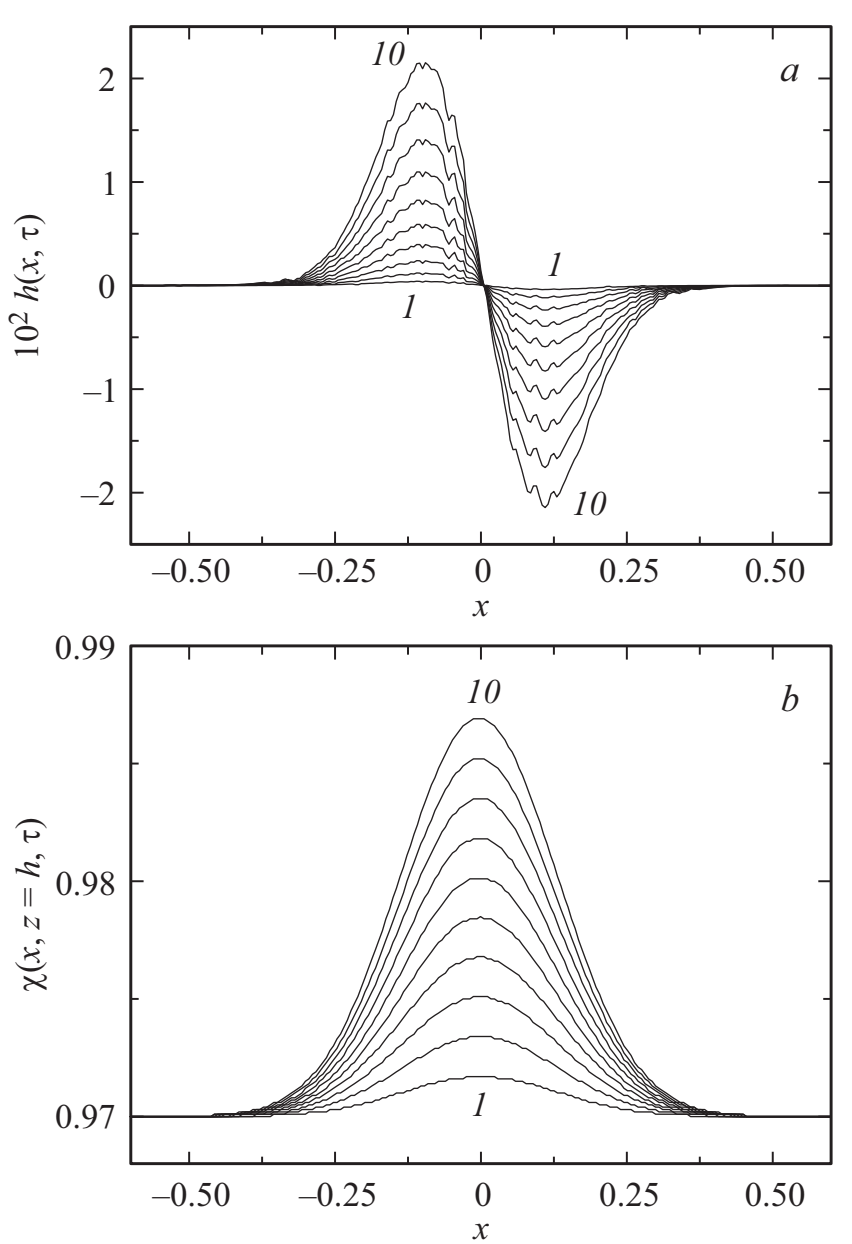

Рис. 1. Эволюция безразмерных профилей деформации свободной поверхности ЖК-фазы $h(x, \tau)(a)$ и температуры $\chi(x, \tau)(b)$ по длине ЖК-канала $-10 \leq x \leq 10$ в процессе его разогрева лазерным лучом мощностью $0.5 \mathrm{~W}$ и продолжительностью $2 \mu \mathrm{s}$, сфокусированным в точке $x_{0}=0.0, z_{0}=0.93$. Результаты даны для 10 значений времени $\tau_{i}=2^{i} \cdot 10^{-5}$ $(i=1, \ldots, 10)$.

$\hat{\mathbf{n}}_{\mathrm{el}}(x, z)$ и температуры $\chi(x, z, \Delta \tau)$, соответствующими моменту времени $\Delta \tau$, было рассчитано распределение функции тока $\psi(x, z, \Delta \tau)$. Следующий шаг по времени $\Delta \tau$ для распределения поля скорости, температуры и поля директора по сечению ЖК-образца со свободной поверхностью был осуществлен с помощью сеточного метода [23], причем устойчивость численной процедуры определялась из условия

$$
\begin{gathered}
\frac{\Delta \tau}{\delta_{3}}\left(\frac{1}{(\Delta x)^{2}}+\frac{1}{(\Delta z)^{2}}\right) \leq \frac{1}{2}, \\
\frac{3 a_{5}}{(\Delta x)^{4}}-\frac{2 a_{1}}{(\Delta z)^{4}}>0,
\end{gathered}
$$

где $\Delta x$ и $\Delta z$ - приращения пространственных переменных, а коэффициенты $a_{1}$ и $a_{5}$ приведены в Приложении. Условием сходимости итерационной процедуры была выбрана величина $\epsilon=\mid\left(\chi_{(m+1)}(x, z, \tau)\right.$ $\left.-\chi_{(m)}(x, z, \tau)\right) / \chi_{(m)}(x, z, \tau) \mid \sim 10^{-4}, \quad$ и $\quad$ итерационная процедура продолжалась вплоть до достижения заданной точности $\epsilon \sim 10^{-4}$. Здесь $m-$ число итераций. На рис. 1, $а$ представлены результаты расчета безразмерного профиля деформации свободной границы раздела ЖК-фаза/воздух $h(x, \tau)=H(x, \tau)-1$ по длине ЖК-канала $-10 \leq x \leq 10$, соответствующие первым 10 значениям времени $\tau_{i}=2^{i} \cdot 10^{-5}(i=1, \ldots, 10)$. При этом время инжекции лазерного излучения мощностью $\mathscr{V}_{0}=0.5 \mathrm{~W}$ и сфокусированного вблизи границы раздела ЖК-фаза/воздух на $\sim 0.7 \mu \mathrm{m}$ вглубь 10 микронной ЖК-фазы было продолжительностью $t_{\text {in }} \sim 2.0 \mu \mathrm{s}$. Расчеты показывают, что в процессе разогрева изначально плоская свободная граница раздела ЖК-фаза/воздух деформируется сильнее всего вблизи точек $x= \pm 0.125$, и приобретает „волнообразный“ профиль, причем максимальная высота гребня „волны“ достигает значения $\left|h\left(x \sim \pm 1, \tau_{\text {in }}=\tau_{10}=0.01\right)\right| \sim 0.02$, т. е., $\sim 2 \%$ от толщины ЖК-слоя. На рис. $1, b$ представлены результаты расчетов эволюции распределения безразмерной температуры $\chi_{z=H}(x, \tau)$ по длине ЖК-канала $-10 \leq x \leq 10$, соответствующие тем же значениям времени что и на рис. 1, $a$. Расчеты свидетельствуют о том, что прогревание ЖК-объема носит локальный характер ( 1.5\% от всего объема ЖК-фазы) и максимальная температура разогрева ЖК-фазы $\chi(x=0.0)_{z=H} \sim 0.987$ $(\sim 307 \mathrm{~K})$ достигается спустя $\tau_{\text {in }}=\tau_{10}=0.01(\sim 2 \mu \mathrm{s})$. Эволюция безразмерных профилей компонент скорости $\mathbf{v}_{z=H}=u_{z=H} \hat{\mathbf{i}}+w_{z=H} \hat{\mathbf{k}} \quad$ на свободной поверхности ЖК-фазы, соответствующих тем же значениям времени что и на рис. $1, a$, представлены на рис. $2, a, b$. Эволюция горизонтальной $u$ и вертикальной $w$ составляющих
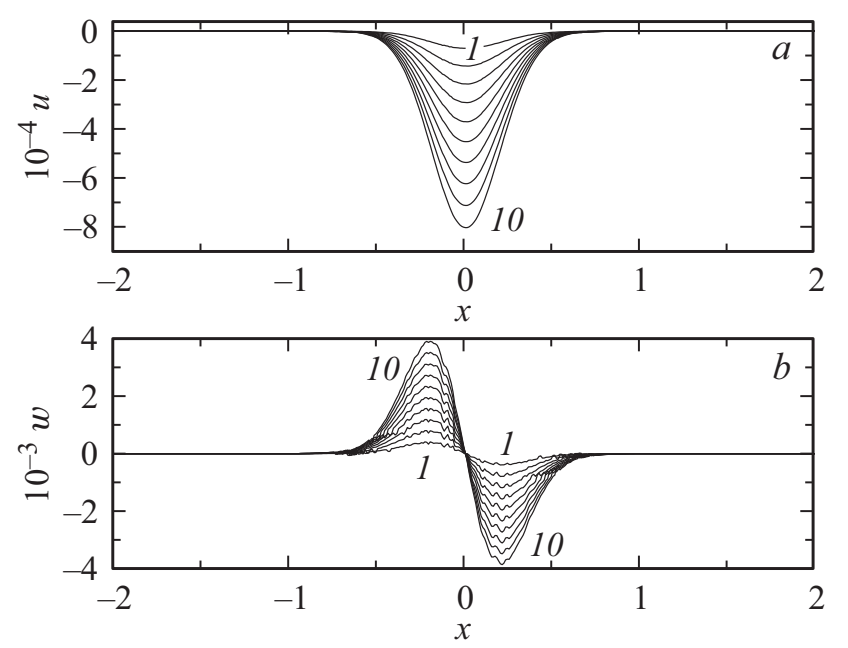

Рис. 2. Эволюция безразмерных профилей горизонтальной $u(x, \tau)_{z=H} \quad(a)$ и вертикальной $w(x, \tau)_{z=H} \quad(b)$ компонент вектора скорости на свободной поверхности ЖК-фазы по длине ЖК-канала $-10 \leq x \leq 10$ в процессе его разогрева лазерным лучом мощностью $0.5 \mathrm{~W}$ и продолжительностью $2 \mu \mathrm{s}$, сфокусированным в точке $x_{0}=0.0, z_{0}=0.93$. Времена те же что на рис. 1. 


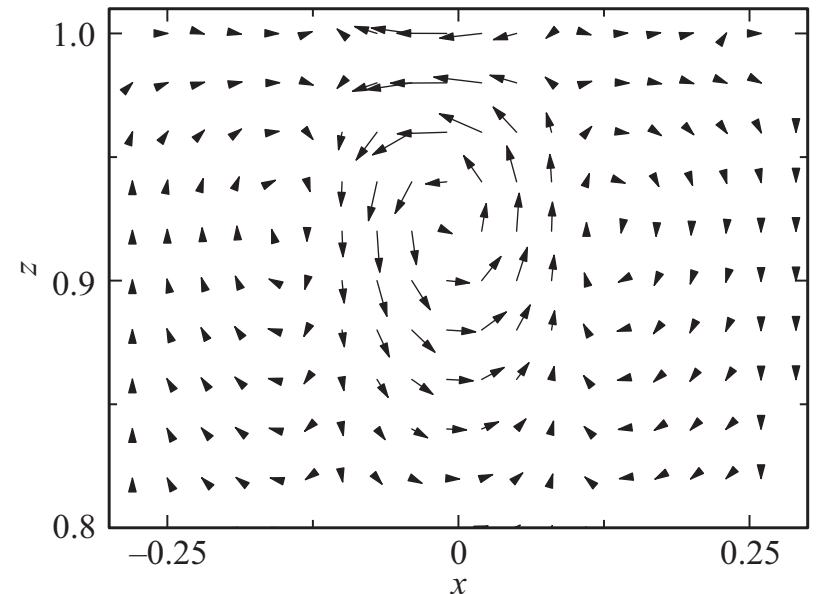

Рис. 3. Распределение поля скорости $\mathbf{v}=u \hat{\mathbf{i}}+w \hat{\mathbf{k}}$ в объеме ЖК-фазы вблизи фокуса лазерного излучения. $1 \mathrm{~mm}$ длины стрелки соответствует $0.4 \mu \mathrm{m} / \mathrm{s}$.

вектора скорости в процессе разогрева ЖК-фазы свидетельствует о том, что вблизи свободной границы формируются три вихря: один крупный, вращающийся против часовой стрелки, и два мелких, вращающихся по часовой стрелке соответственно (см. рис. 3). Координаты центра первого вихря $x=0.0, z=0.93$, в то время как координаты центров двух других вихрей $x= \pm 0.125$, $z=0.93$ соответственно. Расчеты показывают, что найбольшее значение горизонтальной составляющей скорости $u \sim 8 \cdot 10^{-3}(\sim 0.27 \mu \mathrm{m} / \mathrm{s})$ достигается в точке $x=0.0, z \sim 1.0$ и скорость направлена в сторону левого конца ЖК-канала $(x=-10)$, в то время как максимальное значение вертикальной составляющей скорости $w \sim 4 \cdot 10^{-4}(\sim 13.2 \mathrm{~nm} / \mathrm{s})$ направленой в положительном смысле достигается в точке $x=-0.13, z \sim 1.0$, а в отрицательном смысле вблизи точки $x=0.13$, $z \sim 1.0$ соответственно. Расчеты также свидетельствуют о том, что в вихревой поток, создаваемый лазерным излучением мощностью $\mathscr{V}_{0}=0.5 \mathrm{~W}$ и продолжительностью $t_{\text {in }} \sim 2.0 \mu \mathrm{s}$, вовлечена незначительная область ЖК-фазы ограниченная размерами $-0.3 \leq x \leq 0.3$, $0.8 \leq z \leq 1.0$, или $\sim 1.2 \%$ всего объема ЖК-фазы. Следует отметить, что ограничения налагаемые на энергетические параметры лазерного излучения вытекают из небольшого температурного интервала существования нематической фазы образованной молекулами 5ЦБ.

С момента выключения лазерного излучения начинается процесс охлаждения ЖК-фазы $\chi_{z=H}(x, \tau)$ (см. рис. $4, b)$ и, как следствие, уменьшение характера деформации свободной границы раздела ЖК-фаза/воздух $h(x, \tau)$ (см. рис. 4,a), а также затухание вихревого потока (рис. 5, $a, b$ ). На рис. 4-5 показана эволюция вышеописанных характеристик и компонент вектора скорости $u_{z=H}(x, \tau)$ и $w_{z=H}(x, \tau)$ по длине ЖК-канала $-10 \leq x \leq 10$, соответствующих последующим 8 значениям времени $\tau_{i}=2^{i} \cdot 10^{-2}(i=1, \ldots, 8)$. Вихревой поток в ЖК-канале полностью затухает спустя время $\tau_{8} \sim 2.56(\sim 0.5 \mathrm{~s})$ после выключения лазерного излучения. При этом как температурный профиль $\chi(x, \tau)$, так и $h(x, \tau)$ в процессе охлаждения сдвигаются в
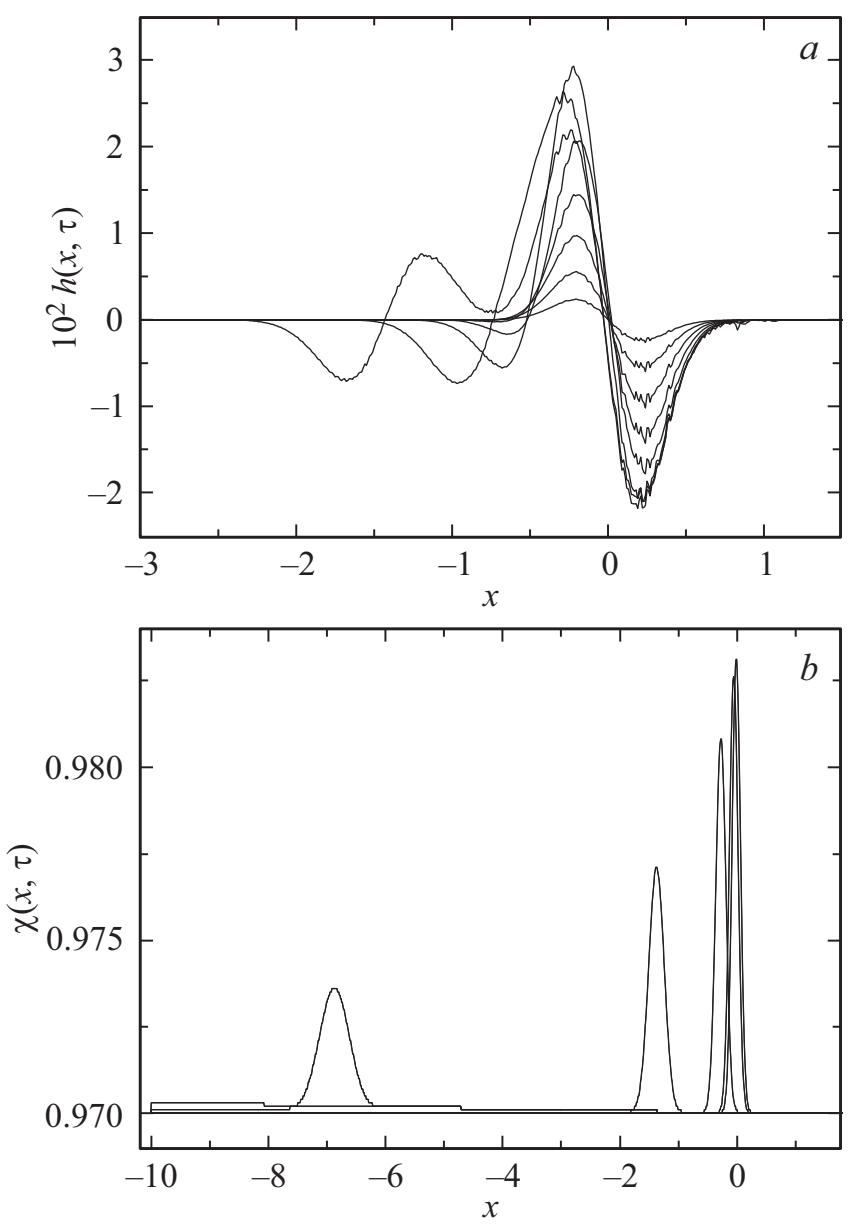

Рис. 4. То же что на рис. 1, но для процесса охлаждения ЖК-фазы. Результаты даны для 8 значений времени $\tau_{i}=2^{i} \cdot 10^{-2}(i=1, \ldots, 8)$.
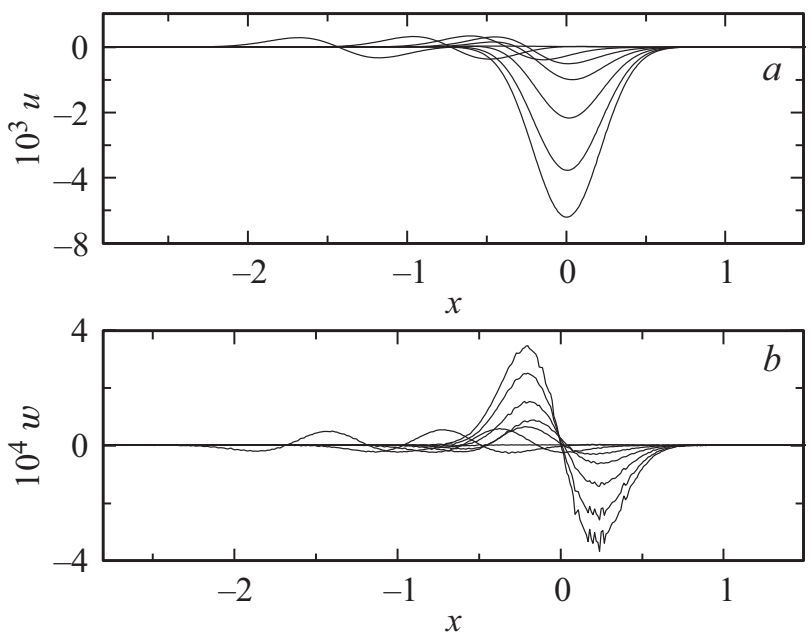

Рис. 5. То же что на рис. 2, но для процесса охлаждения ЖК-фазы. Времена те же что на рис. 4. 

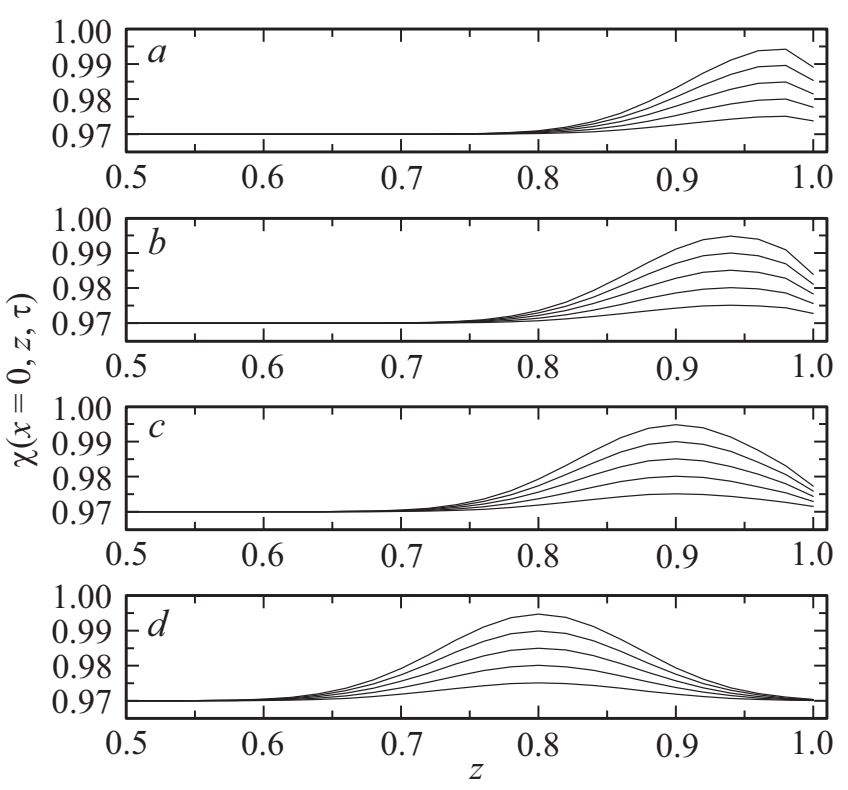

Рис. 6. Распределение значений безразмерной температуры $\chi(x=0.0, z, \tau)$ по сечению ЖК-канала $0.6 \leq z \leq 1.0$ в процессе его разогрева лазерным лучом мощностью $0.5 \mathrm{~W}$ и продолжительностью $2 \mu \mathrm{s}$, сфокусированному в точке $x_{0}=0.0$, а глубина фокусировки варьировалась от значения $z_{0}=0.98(a)$ до $0.94(b)$, и далее до $0.90(c)$, и заканчивая значением $0.80(d)$. Результаты даны для 5 значений времени $\tau_{i}=2^{i} \cdot 10^{-5}$ $(i=6, \ldots, 10)$.

левую сторону ЖК-канала, что вызвано существованием относительно большой горизонтальной составляющей $u$ вектора скорости направленной в сторону левого края ЖК-канала (см. рис. 5,a). На рис. 6 приведено распределение безразмерной температуры $\chi(x=0, z, \tau)$ вдоль вертикальной оси $0.5 \leq z \leq 1.0$, когда лазерный луч мощностью $\mathscr{V}_{0}=0.5 \mathrm{~W}$ и продолжительностью $\tau_{\text {in }}=0.01$ направлен в точку $x_{0}=0.0$. Результаты даны для 5 значений времени $\tau_{i}=2^{i} \cdot 10^{-5}(i=6, \ldots, 10)$. При этом глубина фокусировки лазерного излучения менялась от величины $z_{0}=0.98(a)$ до $z_{0}=0.94(b)$, далее $z_{0}=0.90(c)$, и заканчивая $z_{0}=0.80(d)$ соответственно. Результаты расчетов указывают на слабую зависимость профилей соответствующих распределений температуры по указанной толщине ЖК-материала от глубины фокусировки лазерного излучения. Так, например, для случая $z_{0}=0.80$ мощности лазерного излучения $\mathscr{V}_{0}=0.5 \mathrm{~W}$ не хватает на то, чтобы за время $\tau_{\text {in }}=\tau_{10}=0.01$ прогретая область, где $\chi>\chi_{\text {boundary }}=0.97$, достигла бы свободной поверхности ЖК-фазы. На рис. 7-8 приведены результаты расчета профилей безразмерных горизонтальной $u(x=0, z, \tau)$ (рис. 7) и вертикальной $w(x=0, z, \tau)$ (рис. 8) компонент вектора скорости вдоль вертикальной оси $0.7 \leq z \leq 1.0$, когда лазерный луч мощностью $\mathscr{V}_{0}=0.5 \mathrm{~W}$ направлен в точку $x_{0}=0.0$. Здесь значения времени те же что и на рис. 6. Показано, что по мере того, как фокус лазерного излучения смещает- ся вглубь ЖК-материала, направление горизонтальной компоненты вектора скорости $u(x=0.0, z, \tau)$ меняет знак с отрицательного на положительный, примерно на глубине $z_{0}=0.90$. При этом, направление вертикальной составляющей вектора скорости $w(x=0.0, z, \tau)$ не меняется в течении всех расчетных значений времени $\tau_{i}=2^{i} \cdot 10^{-5}(i=6, \ldots, 10)$ и величина этой скорости

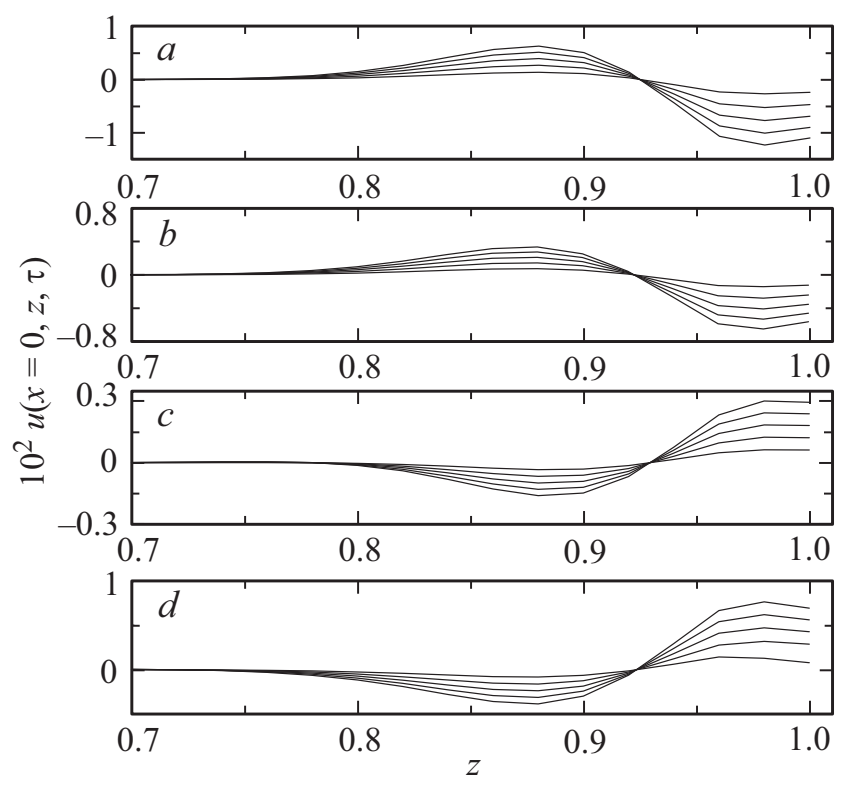

Рис. 7. Распределение безразмерной горизонтальной $u$ $(x=0.0, z, \tau)$ компоненты вектора скорости по сечению ЖК-канала $0.7 \leq z \leq 1.0$ в процессе его разогрева лазерным лучом. Остальные значения те же что на рис. 6.

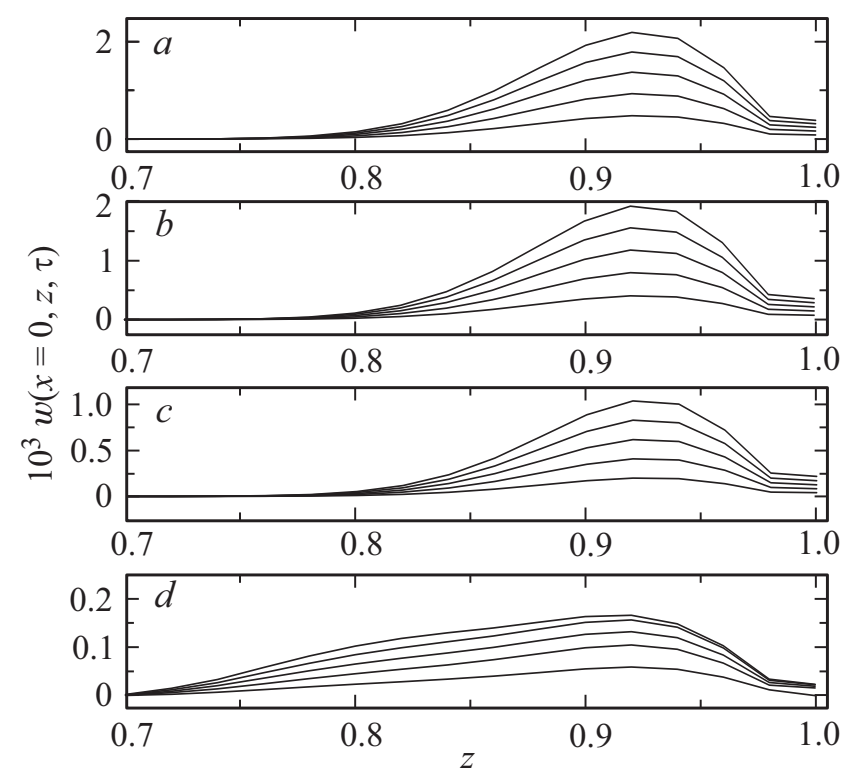

Рис. 8. То же что на рис. 7 , но для распределения безразмерной вертикальной $w(x=0.0, z, \tau)$ компоненты вектора скорости по сечению ЖК-канала $0.7 \leq z \leq 1.0$ в процессе его разогрева лазерным лучом. 
быстро стремится к нулю, по мере приближения к свободной поверхности ЖК-фазы.

Таким образом, расчеты показывают, что изменяя глубину фокусировки лазерного излучения можно добиться изменения направления и величины вихревых потоков в микролитровых ЖК-объемах и, тем самым, добиться необходимой транспортировки ЖК-капель помещенных в микроразмерные каналы или капилляры [5].

\section{4. Заключение}

Анализ полученных результатов касающихся эволюции поля директора, скорости и температуры в ЖК-каналах со свободной поверхностью под действием лазерного излучения показал, что в рамках нелинейного обобщения классической теории Эриксена-Лесли, допускающей учет термомеханических вкладов в выражения для компонент тензора напряжений и баланса моментов сил действующих на единицу объема ЖК-фазы, возможно описание зарождения и формирования вихревых потоков в этих микрометровых ЖК-каналах. Было обнаружено, что в процессе разогрева ЖК-канала лазерным излучением допустимой мощности произошла незначительная переориентация поля директора, повлекшая за собой формирование локального вихревого течения в ЖК-канале. Было также показано, что изменяя глубину фокусировки лазерного излучения можно добиться изменения направления и величины вихревых потоков в микролитровых ЖК-объемах. Все это позволяет надеяться на то, что локальный разогрев ЖК-фазы посредством лазерного излучения является удобным инструментом для транспортировки ЖК-капель помещенных в микроразмерные каналы или капилляры, что указывает на то, что существует возможность немеханической транспортировки или сортировки микролитровых объемов ЖК-фазы под действием сфокусированного лазерного излучения.

Исследованные в работе особенности, связанные с реакцией ЖК-материалов на локализованное воздействие градиента температуры и градиента поля директора, необходимо учитывать при создании разнообразных сенсоров и датчиков используемых в биотехнологических приложениях, медицине и фармокологии, а также в иммерсионной литографии.

\section{5. Приложение}

Безразмерные компоненты тензора напряжений имеют вид

$$
\begin{aligned}
\sigma_{x x}^{\mathrm{el}} & =\delta_{1}\left[-n_{x, x} \mathscr{M}_{0}+K_{31} f n_{z, x}\right], \\
\sigma_{z z}^{\mathrm{el}} & =\delta_{1}\left[-n_{x, x} \mathscr{M}_{0}+K_{31} f n_{z, z}\right], \\
\sigma_{x z}^{\mathrm{el}} & =\delta_{1}\left[-n_{x, x} \mathscr{M}_{0}-K_{31} f n_{x, x}\right], \\
\sigma_{z x}^{\mathrm{el}} & =\delta_{1}\left[-n_{x, x} \mathscr{M}_{0}-K_{31} f n_{x, z}\right],
\end{aligned}
$$

$$
\begin{aligned}
\sigma_{x x}^{\mathrm{vis}}= & \gamma_{21} n_{x} \frac{d n_{x}}{d t}+\psi_{, x z} \frac{1}{\gamma_{1}}\left[\alpha_{4}+\left(\alpha_{5}+\alpha_{6}\right) n_{x}^{2}\right. \\
& \left.+\alpha_{1} n_{x}^{2}\left(n_{x}^{2}-n_{z}^{2}\right)\right]+\frac{\alpha_{1}}{\gamma_{1}} n_{x}^{3} n_{z}\left(\psi_{, z z}-\psi_{, x x}\right) \\
& -\frac{1}{2} \gamma_{21} n_{x} n_{z}\left(\psi_{, z z}+\psi_{, x x}\right)
\end{aligned}
$$

$$
\begin{aligned}
\sigma_{x z}^{\mathrm{vis}}= & \frac{1}{2}\left(n_{z} \frac{d n_{x}}{d t}-n_{x} \frac{d n_{z}}{d t}\right)+\frac{1}{2} \gamma_{21}\left(n_{z} \frac{d n_{x}}{d t}+n_{x} \frac{d n_{z}}{d t}\right) \\
& +\psi_{, x z} \frac{1}{\gamma_{1}}\left[\alpha_{1} n_{x} n_{z}\left(n_{x}^{2}-n_{z}^{2}\right)+\gamma_{2} n_{x} n_{z}\right] \\
& +\frac{1}{\gamma_{1}}\left(\psi_{, z z}-\psi_{, x x}\right)\left[\alpha_{1} n_{x}^{2} n_{z}^{2}+\frac{\alpha_{4}}{2}+\frac{\alpha_{5}+\alpha_{6}}{4}\right] \\
& -\frac{1}{4}\left(\psi_{, z z}+\psi_{, x x}\right)+\frac{1}{2} \gamma_{21} \psi_{, x x}\left(n_{x}^{2}-n_{z}^{2}\right),
\end{aligned}
$$$$
\sigma_{z x}^{\mathrm{vis}}=\frac{1}{2}\left(n_{x} \frac{d n_{z}}{d t}-n_{z} \frac{d n_{x}}{d t}\right)+\frac{1}{2} \gamma_{21}\left(n_{z} \frac{d n_{x}}{d t}+n_{x} \frac{d n_{z}}{d t}\right)
$$$$
-\psi_{, x z} \frac{1}{\gamma_{1}}\left[\alpha_{1} n_{x} n_{z}\left(n_{z}^{2}-n_{x}^{2}\right)+\gamma_{2} n_{x} n_{z}\right]
$$$$
+\frac{1}{\gamma_{1}}\left(\psi_{, z z}-\psi_{, x x}\right)\left[\alpha_{1} n_{x}^{2} n_{z}^{2}+\frac{\alpha_{4}}{2}+\frac{\alpha_{5}+\alpha_{6}}{4}\right]
$$$$
+\frac{1}{4}\left(\psi_{, z z}+\psi_{, x x}\right)+\frac{1}{2} \gamma_{21} \psi_{, z z}\left(n_{x}^{2}-n_{z}^{2}\right),
$$

$$
\begin{aligned}
\sigma_{z z}^{\mathrm{vis}}= & \gamma_{21} n_{z} \frac{d n_{z}}{d t}-\psi_{, x z} \frac{1}{\gamma_{1}}\left[\alpha_{4}+\left(\alpha_{5}+\alpha_{6}\right) n_{z}^{2}+\alpha_{1} n_{z}^{2}\left(n_{z}^{2}-n_{x}^{2}\right)\right] \\
+ & \alpha_{1} n_{z}^{3} n_{x}\left(\psi_{, z z}-\psi_{, x x}\right)+\frac{1}{2} \gamma_{21} n_{x} n_{z}\left(\psi_{, z z}+\psi_{, x x}\right) \\
\sigma_{x x}^{\mathrm{tm}}= & \delta_{1} \chi_{, x}\left[\frac{1}{2} M_{x x} n_{x}\left(3 n_{x}^{2}+7\right) M_{x z} n_{z}\left(1+\frac{7}{2} n_{x}^{2}\right)\right. \\
& \left.+M_{z z} n_{x}\left(\frac{3}{2}+2 n_{z}^{2}\right)\right]+\delta_{1} \chi_{, z}\left[M_{x x} n_{z}\left(n_{x}^{2}+1\right)\right. \\
& \left.+\frac{1}{2} M_{x z} n_{x}\left(3+5 n_{z}^{2}\right)+\frac{3}{2} M_{z z} n_{z}\left(1+n_{z}^{2}\right)\right]
\end{aligned}
$$

$$
\begin{aligned}
& \sigma_{x z}^{\mathrm{tm}}=\delta_{1} \chi_{, x}\left[\frac{1}{2} M_{x x} n_{z}\left(n_{x}^{2}+3\right)+M_{x z} n_{x}\left(3 n_{z}^{2}-\frac{1}{2}\right)+M_{z z} n_{z}^{3}\right] \\
& +\delta_{1} \chi_{, z}\left[\frac{1}{2} M_{x x} n_{x}-\frac{1}{2} M_{x z} n_{z}\left(3-n_{x}^{2}\right)-\frac{1}{2} M_{z z} n_{x} n_{z}^{2}\right], \\
& \sigma_{z x}^{\mathrm{tm}}=\delta_{1} \chi_{, x}\left[-\frac{1}{2} M_{x x} n_{z} n_{x}^{2}+\frac{1}{2} M_{x z} n_{x}\left(3-n_{z}^{2}\right)+\frac{1}{2} M_{z z} n_{z}\right] \\
& +\delta_{1} \chi_{, z}\left[M_{x x} n_{x}^{3}+\frac{1}{2} M_{x z} n_{z}\left(3 n_{x}^{2}-1\right)+\frac{1}{2} M_{z z} n_{x}\left(1+n_{z}^{2}\right)\right],
\end{aligned}
$$




$$
\begin{aligned}
\sigma_{z z}^{\mathrm{tm}}= & \delta_{1} \chi_{, x}\left[\frac{3}{2} M_{x x} n_{x}\left(n_{x}^{2}+1\right)+M_{x z} n_{z}\left(3+5 n_{x}^{2}\right)\right. \\
& \left.+M_{z z} n_{x}\left(1+n_{z}^{2}\right)\right]+\delta_{1} \chi_{, z}\left[M_{x x} n_{z}\left(2 n_{x}^{2}+\frac{3}{2}\right)\right. \\
& \left.+M_{x z} n_{x}\left(1+\frac{7}{2} n_{z}^{2}\right)+\frac{1}{2} M_{z z} n_{z}\left(7+3 n_{z}^{2}\right)\right]
\end{aligned}
$$

Коэффициенты $a_{i}(i=1, \ldots 12)$ уравнения (11) имеют вид

$$
\begin{aligned}
a_{1}= & \frac{1}{2}+\frac{1}{\gamma_{1}}\left[\frac{\alpha_{4}}{2}+\frac{\alpha_{5}+\alpha_{6}}{4}+\alpha_{1} n_{x}^{2} n_{z}^{2}\right] \\
& +\frac{\gamma_{21}}{2}\left(n_{x}^{2}-n_{z}^{2}\right)-\frac{\gamma_{21}^{2}}{4}\left(n_{x}^{2}-n_{z}^{2}\right)^{2},
\end{aligned}
$$

$a_{2}=-\frac{2}{\gamma_{1}}\left[\alpha_{1} n_{x} n_{z}\left(n_{z}^{2}-n_{x}^{2}\right)+\gamma_{2} n_{x} n_{z}\right]+2 \gamma_{21}^{2} n_{x} n_{z}\left(n_{x}^{2}-n_{z}^{2}\right)$,

$$
\begin{aligned}
a_{3}= & 1+\frac{1}{\gamma_{1}}\left[\alpha_{4}+\frac{\alpha_{5}+\alpha_{6}}{2}-\alpha_{1}\left(n_{x}^{4}+n_{z}^{4}\right)\right] \\
& -4 \gamma_{21}^{2} n_{x}^{2} n_{z}^{2}+\frac{1}{2} \gamma_{21}^{2}\left(n_{x}^{2}-n_{z}^{2}\right),
\end{aligned}
$$

$a_{4}=\frac{2}{\gamma_{1}}\left[\alpha_{1} n_{x} n_{z}\left(n_{z}^{2}-n_{x}^{2}\right)-\gamma_{2} n_{x} n_{z}\right]-2 \gamma_{21}^{2} n_{x} n_{z}\left(n_{x}^{2}-n_{z}^{2}\right)$,

$$
\begin{aligned}
a_{5}= & \frac{1}{2}+\frac{1}{\gamma_{1}}\left[\frac{\alpha_{4}}{2}+\frac{\alpha_{5}+\alpha_{6}}{4}+\alpha_{1} n_{x}^{2} n_{z}^{2}\right]^{2} \frac{\gamma_{21}}{2}\left(n_{x}^{2}-n_{z}^{2}\right)-\frac{\gamma_{21}^{2}}{4}\left(n_{x}^{2}-n_{z}^{2}\right)^{2}, \\
a_{6}= & \frac{1}{\gamma_{1}}\left[\left(\alpha_{1}+\frac{\gamma_{2}^{2}}{\gamma_{1}}\right) n_{x} n_{z}\left(n_{x}^{2}-n_{z}^{2}\right)+2 \gamma_{2} n_{x} n_{z}\right]_{, x} \\
+ & \frac{1}{\gamma_{1}}\left[\alpha_{1} n_{x}^{2} n_{z}^{2}+\frac{\gamma_{2}}{2}\left(n_{x}^{2}-n_{z}^{2}\right)\right]_{, z}-\frac{\gamma_{21}^{2}}{4}\left[\left(n_{x}^{2}-n_{z}^{2}\right)^{2}\right]_{, z}, \\
a_{7}= & -\frac{2}{\gamma_{1}}\left[\alpha_{1} n_{x} n_{z}\left(n_{x}^{2}-n_{z}^{2}\right)+\gamma_{2} n_{x} n_{z}\right]_{, z} \\
& -\frac{1}{\gamma_{1}}\left[\alpha_{1}\left(n_{x}^{2}-n_{z}^{2}\right)^{2}+4 \frac{\gamma_{2}^{2}}{\gamma_{1}} n_{x}^{2} n_{z}^{2}\right]_{, x} \\
+ & \frac{1}{4}\left[1+\gamma_{21}\left(n_{x}^{2}-n_{z}^{2}\right)\right]_{, x}^{2}+2 \gamma_{21}^{2}\left[n_{x} n_{z}\left(n_{x}^{2}-n_{z}^{2}\right)\right]_{, z}, \\
a_{8}= & \frac{1}{\gamma_{1}}\left[\left(-\alpha_{1}+\frac{\gamma_{2}^{2}}{4 \gamma_{1}}\right)\left(n_{z}^{2}-n_{x}^{2}\right)^{2}-\left(2 \alpha_{1}+\frac{4 \gamma_{2}^{2}}{\gamma_{1}}\right) n_{x}^{2} n_{z}^{2}\right]_{, z} \\
+ & \frac{2}{\gamma_{1}}\left[\left(\alpha_{1}+\frac{\gamma_{2}^{2}}{\gamma_{1}}\right)\left(n_{z}^{2}-n_{x}^{2}\right) n_{x} n_{z}-\gamma_{2} n_{x} n_{z}\right]_{, x}, \\
a_{9}= & \frac{1}{\gamma_{1}}\left[-\left(\alpha_{1}+\frac{\gamma_{2}^{2}}{\gamma_{1}}\right) n_{x} n_{z}\left(n_{x}^{2}-n_{z}^{2}\right)-2 \gamma_{2} n_{x} n_{z}\right]_{, z} \\
+ & \frac{1}{\gamma_{1}}\left[\alpha_{1} n_{x}^{2} n_{z}^{2}-\frac{\gamma_{2}}{2}\left(n_{x}^{2}-n_{z}^{2}\right)\right]_{, x}-\frac{\gamma_{21}^{2}}{4}\left[\left(n_{x}^{2}-n_{z}^{2}\right)^{2}\right]_{, x},
\end{aligned}
$$

$$
\begin{aligned}
& a_{10}=\frac{1}{\gamma_{1}}\left[\alpha_{1} n_{x}^{2} n_{z}^{2}+\frac{3 \gamma_{2}}{4}\left(n_{x}^{2}-n_{z}^{2}\right)\right]_{, z z} \\
& -\frac{1}{\gamma_{1}}\left[\alpha_{1} n_{x}^{2} n_{z}^{2}+\frac{\gamma_{2}}{4}\left(n_{x}^{2}-n_{z}^{2}\right)\right]_{, x x}+\frac{\gamma_{21}}{4}\left(n_{x}^{2}-n_{z}^{2}\right)\left[\left(n_{x}^{2}-n_{z}^{2}\right)_{, x x}\right. \\
& \left.-2\left(n_{x}^{2}-n_{z}^{2}\right)_{, z z}\right]-\left[\frac{\alpha_{1}}{\gamma_{1}} n_{x} n_{z}\left(n_{z}^{2}-n_{x}^{2}\right)-\gamma_{21} n_{x} n_{z}\right]_{, x z} \\
& +\gamma_{21}^{2} n_{x} n_{z}\left(n_{x}^{2}-n_{z}^{2}\right)_{, x z}+\gamma_{21}^{2}\left[\left(n_{x} n_{z}\right)_{, x}\left(n_{x}^{2}-n_{z}^{2}\right)_{, z}\right. \\
& \left.+\left(n_{x} n_{z}\right)_{, z}\left(n_{x}^{2}-n_{z}^{2}\right)_{, x}\right] \\
& -\gamma_{21}^{2}\left[\frac{1}{4}\left(\left(n_{x}^{2}-n_{z}^{2}\right)_{, z}\right)^{2}-\frac{1}{4}\left(\left(n_{x}^{2}-n_{z}^{2}\right)_{, x}\right)^{2}\right], \\
& a_{11}=-\frac{1}{\gamma_{1}}\left[\alpha_{1} n_{x} n_{z}\left(n_{x}^{2}-n_{z}^{2}\right)\right]_{, x x}-\frac{1}{\gamma_{1}}\left[\alpha_{1} n_{x} n_{z}\left(n_{x}^{2}-n_{z}^{2}\right)\right]_{, z z} \\
& -\frac{\alpha_{1}}{\gamma_{1}}\left[\left(n_{x}^{2}-n_{z}^{2}\right)^{2}\right]_{, x z}-\gamma_{21}^{2}\left[n_{x} n_{z}\left(n_{z}^{2}-n_{x}^{2}\right)_{, z z}+\left(n_{x} n_{z}\right)_{, z z}\right. \\
& \left.\times\left(n_{x}^{2}-n_{z}^{2}\right)\right] \gamma_{21}^{2}\left[-n_{x} n_{z}\left(n_{z}^{2}-n_{x}^{2}\right)_{, x x}+\left(n_{x} n_{z}\right)_{, x x}\left(n_{x}^{2}-n_{z}^{2}\right)\right] \\
& +\gamma_{21}^{2}\left[8 n_{x} n_{z}\left(n_{z} n_{x}\right)_{, x z}+8\left(n_{z} n_{x}\right)_{, z}\left(n_{z} n_{x}\right)_{, x}\right. \\
& \left.+\left(n_{z} n_{x}\right)_{, z}\left(n_{x}^{2}-n_{z}^{2}\right)_{, x}\right]+\gamma_{21}^{2}\left[\left(n_{z} n_{x}\right)_{, x}\left(n_{x}^{2}-n_{z}^{2}\right)_{, x}\right], \\
& a_{12}=\frac{1}{\gamma_{1}}\left[\alpha_{1} n_{x}^{2} n_{z}^{2}-\frac{3 \gamma_{2}}{4}\left(n_{x}^{2}-n_{z}^{2}\right)\right]_{, x x} \\
& +\frac{1}{\gamma_{1}}\left[\alpha_{1} n_{x}^{2} n_{z}^{2}+\frac{\gamma_{2}}{4}\left(n_{x}^{2}-n_{z}^{2}\right)\right]_{, z z}+\frac{\gamma_{21}^{2}}{4}\left(n_{x}^{2}-n_{z}^{2}\right)\left[\left(n_{x}^{2}-n_{z}^{2}\right)_{, z z}\right. \\
& \left.-2\left(n_{x}^{2}-n_{z}^{2}\right)_{, x x}\right]-\left[\frac{\alpha_{1}}{\gamma_{1}} n_{x} n_{z}\left(n_{z}^{2}-n_{x}^{2}\right)-\gamma_{21} n_{x} n_{z}\right]_{, x z} \\
& -\gamma_{21}\left(n_{x} n_{z}\right)_{, x z}\left[1+\gamma_{21}\left(n_{x}^{2}-n_{z}^{2}\right)\right]-\gamma_{21}^{2} n_{x} n_{z}\left(n_{x}^{2}-n_{z}^{2}\right)_{, x z} \\
& -\gamma_{21}^{2}\left[\left(n_{x} n_{z}\right)_{, z}\left(n_{x}^{2}-n_{z}^{2}\right)_{, x}+\left(n_{x} n_{z}\right)_{, x}\left(n_{x}^{2}-n_{z}^{2}\right)_{, x}\right] \\
& -\frac{\gamma_{21}^{2}}{4}\left[\left(\left(n_{x}^{2}-n_{z}^{2}\right)_{, z}\right)^{2}-\left(\left(n_{x}^{2}-n_{z}^{2}\right)_{, x}\right)^{2}\right] .
\end{aligned}
$$

Матрица $\hat{\mathscr{B}}$ и вектор $\mathbf{C}$ имеют следующие элементы $b_{i j}$ $(i, j=1,2,3)$ и $c_{i}(i=1,2,3)$ :

$$
\begin{gathered}
b_{11}=\left(n_{x}\right)_{z=H}\left(2 \gamma_{21}-\gamma_{21}^{2}+\frac{2 \alpha_{4}+\alpha_{5}+\alpha_{6}-2 \alpha_{1}}{2 \gamma_{1}}\right), \\
b_{12}=-\frac{\alpha_{4}+\alpha_{5}+\alpha_{6}+\alpha_{1}}{\gamma_{1}}, \\
b_{13}=\left(n_{x}\right)_{z=H}\left(\gamma_{21}+\gamma_{21}^{2}-\frac{2 \alpha_{4}+\alpha_{5}+\alpha_{6}+2 \alpha_{1}}{2 \gamma_{1}}\right), \\
b_{21}=\frac{1}{4}-\frac{2 \alpha_{4}+\alpha_{5}+\alpha_{6}}{4 \gamma_{1}}, \\
b_{22}=\left(n_{x}\right)_{z=H}\left(-\gamma_{21}^{2}-\frac{2 \alpha_{4}+\alpha_{5}+\alpha_{6}+2 \alpha_{1}}{\gamma_{1}}\right),
\end{gathered}
$$




$$
\begin{gathered}
b_{23}=\frac{1}{2}-\frac{\gamma_{21}}{2}-\frac{\gamma_{21}^{2}}{4}+\frac{2 \alpha_{4}+\alpha_{5}+\alpha_{6}}{4 \gamma_{1}}, \\
b_{31}=\frac{\gamma_{21}-1}{2}, \\
b_{32}=-2 \gamma_{21}\left(n_{x}\right)_{z=H}, \\
b_{33}=-\frac{\gamma_{21}+1}{2}, \\
c_{1}=2 \gamma\left(n_{x, x}\right)_{z=H}-\mathscr{P}, \\
c_{2}=\frac{\delta_{1}}{2}\left(\gamma_{21}-1\right)\left[3 \chi_{, x}\left(n_{x, x}\right)_{z=H}+\chi_{, z}\left(n_{x, z}\right)_{z=H}\right], \\
c_{3}=\delta_{1}\left(\frac{3}{2} \chi_{, x}\left(n_{x, x}\right)_{z=H}+\chi_{, z}\left(n_{x, z}\right)_{z=H}\right) .
\end{gathered}
$$

Здесь $\mathscr{P}=\frac{d^{2}}{K_{1}} P-$ безразмерное гидростатическое давление в ЖК-системе.

\section{Список литературы}

[1] W. Sparreboom, A. van den Berg, J.C.T. Eijkel. New J. Phys. 12, 0115004 (2010).

[2] T.M. Squires, S.R. Quake. Rev. Mod. Phys. 77, 977 (2005).

[3] E. Verneuil, M.L. Cordero, F. Gallaire, Ch.N. Baroud. Langmuir 25, 5127 (2009).

[4] A.V. Zakharov, A.A. Vakulenko. J. Chem. Phys. 127, 084907 (2007).

[5] A.V. Zakharov, A.A. Vakulenko, Mitsumasa Iwamoto. J. Chem. Phys. 132, 224906 (2010).

[6] S.J. Woltman, G.D. Jay, G.P. Crawford. Nature Mat. 6, 929 (2007).

[7] S. Zhou, A. Sokolov, O.D. Lavrentovich, I.S. Aranson. P.N.A.S. 111, 1265 (2011).

[8] H. Choi, H. Takezoe. Soft Matter. 12, 481 (2016).

[9] A.V. Zakharov, A.A. Vakulenko. Phys. Fluids 27, 062001 (2015).

[10] Р.С. Акопян, Б.Я. Зельдович. ЖЭТФ 87, 1660 (1984).

[11] P.G. de Gennes, J. Prost. The physics of liquid crystals. Oxford Univ. Press, Oxford (1995). 400 p.

[12] J.L. Ericksen. Arch. Ration. Mech. Anal. 4, 231 (1960).

[13] F.M. Leslie. Arch. Ration. Mech. Anal. 28, 265 (1968).

[14] С. Гроот, П. Мазур. Неравновесная термодинамика. Мир, M. (1964). 456 c.

[15] N.V. Madhusudana, R.B. Ratibha. Mol. Cryst. Liq. Cryst. 132, 339 (1986).

[16] A.G. Chmielewski. Mol. Cryst. Liq. Cryst. 132, 339 (1986).

[17] M. Marinelli, A.K. Ghosh, F. Mercury. Phys. Rev. E 63, 061713 (2001).

[18] P. Jamee, G. Pitsi, J. Thoen. Phys. Rev. E 66, 021707 (2002).

[19] A.V. Zakharov, A.A. Vakulenko. Phys. Rev. E 86, 031701 (2012).

[20] I.-C. Khoo, S.-T. Wu, Optics and Nonlinear Optics of Liquid Crystal. World Scientific, Singapure (1993). P. 59.

[21] R.S. Akopyan, R.B. Alaverdian, E.A. Santrosian, Y.S. Chilingarian. J. Appl. Phys. 90, 3371 (2001).

[22] И.С. Березин, Н.Р. Жидков. Методы вычислений. Физматгиз, М. (1964). 464 с.

[23] А.А. Самарский, Е.С. Николаев. Методы решения сеточных уравнений. Наука, М. (1978) 592 с.

Редактор Ю.Э. Китаев 